\title{
Tratamiento de la coartación aórtica en el niño mediante plastía con arteria subclavia
}

\author{
Dra. Inés Araneda A. ${ }^{1 *} ;$ Dr. Oscar Gómez V.2; D.. Claudio A retz V.2; \\ Dr. Luis León M.2; Dr. Fernando Eimbcke M.2 ; Dr. Raúl Zilleruelo B. ${ }^{2}$
}

\section{Aortic coarctation: repair by subclavian artery flap}

\begin{abstract}
Six ty five partient 8 with aortic coarctation were submitted to aortoplasty with left subclavian artery flap between November 1977 and January 1985 . Their age range at surgical repair was 7 days to 10 years and $58 \%$ of them had another associated cardiac malformation. The operative mortality was $3 \%$ and it was always due to respiratory complications. Recoarctation occured in only two patients, both were less than 3 months old at the time of operation. Muscular attophy and slower growth rate of the left upper limb were detected in only one patient in this series. This surgical procedure is our first choice in pediatric patients with coarctation of the aorta because of its low mortality rate and few late complications.
\end{abstract}

(Key words: Aortic coartation, surgical repair, subclavian attery flap).

La coartación aórtica (Co Ao) representa $5 \%$ a $10 \%$ de todas las malformaciones congénitas cardiovasculares. Si bien su corrección quirúrgica debe ser hecha idealmente entre los 2 y 4 años de edad, el defecto suele producir en el lactante insuficiencia cardíaca de difícil manejo médico, obligando a intervenir antes de las edades señaladasi,2. La reparación quirúrgica empleando resección del segmento coartado $y$ anastomosis término-terminal tiene, antes de la edad de 2 años, el inconveniente de alta incidencia de recoartación $(20 \text { a } 35 \%)^{3,4}$. Por esta razón se ha propuesto usar una técrica de plastía aórtica mediante un colgajo de arteria subclavia izquierda obtenido después de seccionar distalmente esta última y abrirla en canal ${ }^{5,6.7}$ (Fig.). Con esta técrica se consigue aumentar el lumen aórtico sin usar elementos extraños, evitando al mismo tiempo las estrecheces residuales que suelen ocurrir en las anastomosis perpendiculares o término-terminales ${ }^{5}$.

Este informe se refiere a los resultados clíni$\cos$ y seguimiento de 65 nitos cuya Co Ao fue reparada mediante plastía de subclavia en los últimos 8 años.

1. Unidad de Cardiología. Servicio de Pediatría. Hospital Exequiel Gonzàlez Cortés.

2. Centro Cardiovascular. Hospital Luís Calvo Mackenna.

* Trabajo de incorporación a la Sociedad Chilena de Pediatría.

\section{MATERIAL Y METODO}

Se analizaron retrospectivamente los antecedentes de 65 pacientes operados entre Noviembre de 1977 y Enero de 1985 por coartación de aorta, mediante plastia con arteria subclavia; 44 eran varones (68\%) y en el momento de la intevención, la edad de los pacjentes fluctuaba entre 7 dias y 10 años, promedio 1 año 2 meses y mediana cinco meses; $70 \%$ de ellos tenían menos de 1. año.

Cuarenta y tres pacientes $(66 \%)$ tenían desnutrición de grado I ó Il (Sempé), 16 (25\%) eran eutróficos, 32 (50\%) pesaban menos de $5 \mathrm{~kg}$ y sólo 5 ( $8 \%$ más de is $\mathrm{kg}$ al ser interveridos (Tabla 1).

Cuarenta y cinco $(69 \%)$ pacientes tenían Co Ao preductal, el resto post ductal del tipo clásico. En 37 (58\%) habían malformaciones cardiacas asociadas; comunicación interventricular (CIV) en 13 (20\%); ductus en 11 (16\%) hipoplasia del istmo y ClV en 2 (3\%) y, en un paciente en cada caso, transposición con CIV; ductus; tron co tipo I; comunicación interauricular (CIA) con CIV y ductus $(n=4)$.

Fl diagnóstico se fundamentó en ausencia o disminución de los pulsos femorales e hipertensión arterial de distintos grados en ias extremidades superiores. Todos los pacientes fueron estudiados con radiografía de tórax y electrocardiograma. La ecocardiografía sólo fue empleada en los últimos 3 años, en 20 pacientes. El estudio hemodinámico fue considerado en 25 pacientes, por tener evidencias de anomalías agregadas. Todos los casos fueron confimados eл la cirugía.

F. 63 pacientes $(96 \%$ se observó disminución o ausencia de pulsos femorales, en 56 (86\%) insuficiencia cardíaca y en $55(85 \%)$ hipertensión arterial ${ }^{14,15,16}$. Se inđicó la intervención en lactantes si existía hipertensión arterial sostenida, insuficiencia cardíaca refractaria al tratamiento con diuréticos y digitálicos o ambos. Los demás pacientes fueron operados entre los 2 y 4 años, 0 después de estas edades cuando la consulta fue tardía. Todos fueron intervenidos con la técnica anteriormente descrita usando oclusión aórtica. 
En 31 pacientes (48\%) se realizaran otros procedimientos adicionales que consistieron en 25 ligaduras y secciones de ductus (sólo 11 habían dado evidencia clínica pre-operatoria) y en $6(9 \%)$ reducción con cinta del calibre de la arteria pulmonar ("banding") por CIV de gran flujo con hipertensión pulmonar.

\section{RESULTADOS}

La cirugía se realizó sin mayores complicaciones. Sólo un caso que presentó hemorragia del sitio de sutura arterial, debió ser reintervenido en el mismo dia, sin consecuencias posteriores.

En el post-operatorio inmediato la complicación más frecuente fue la hipertensión arterial transitoria que ocurrió en 28 casos $(48 \%)$, desa-, parición espontáneamente en 1 a 10 dias y fue tratada con reserpina en 22 pacjentes $y$ diazóxido en 6 , por pequeños periodos de tiempo. Esta complicación afectó a $8 / 26(31 \%)$ niños operados en el primer trimestre de la vida;
Tabla 1.

Fdad y estado de nutrición del nin̄o con Co Ao operada con plastía de subclavia

\begin{tabular}{lrl}
\hline Edad & n & $\%$ \\
\hline $0-3$ mleses & 26 & 40 \\
$3-6$ meses & 13 & 20 \\
$6-12$ meses & 6 & 10 \\
$1 \quad 5$ años & 10 & 15 \\
$\quad 5$ años & 10 & 15
\end{tabular}

Nutrición:

\begin{tabular}{lrr}
\hline Eutrófico & 16 & 25 \\
$\mathrm{D}_{1}$ & 26 & 40 \\
$\mathrm{D}_{2}$ & 17 & 26 \\
$\mathrm{D}_{3}$ & 6 & 9 \\
\hline
\end{tabular}

$8 / 16(50 \%)$ en el segundo trimestre, a todos $6 / 6$ de los operados en el segundo semestre, pero sólo a $5 / 20(25 \%)$ sujetos mayores de 12 meses.

Las infecciones respiratorias afectaron a 12
A

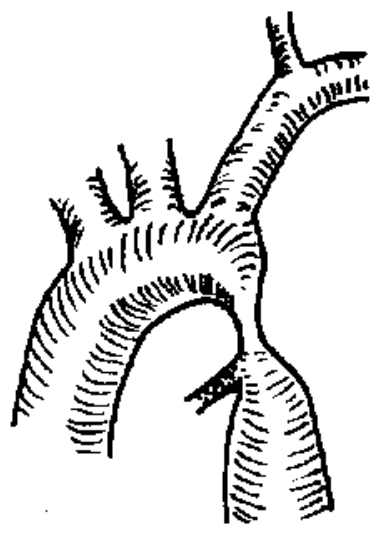

B

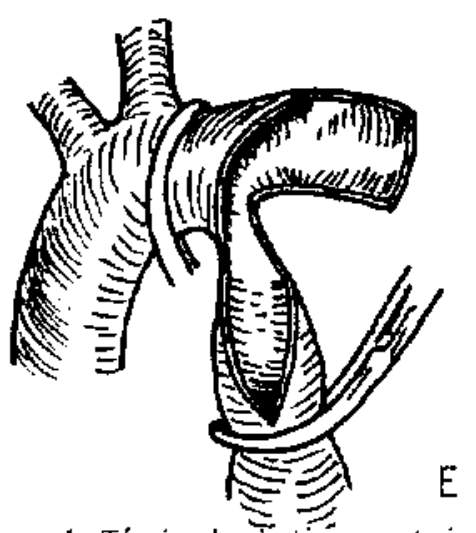

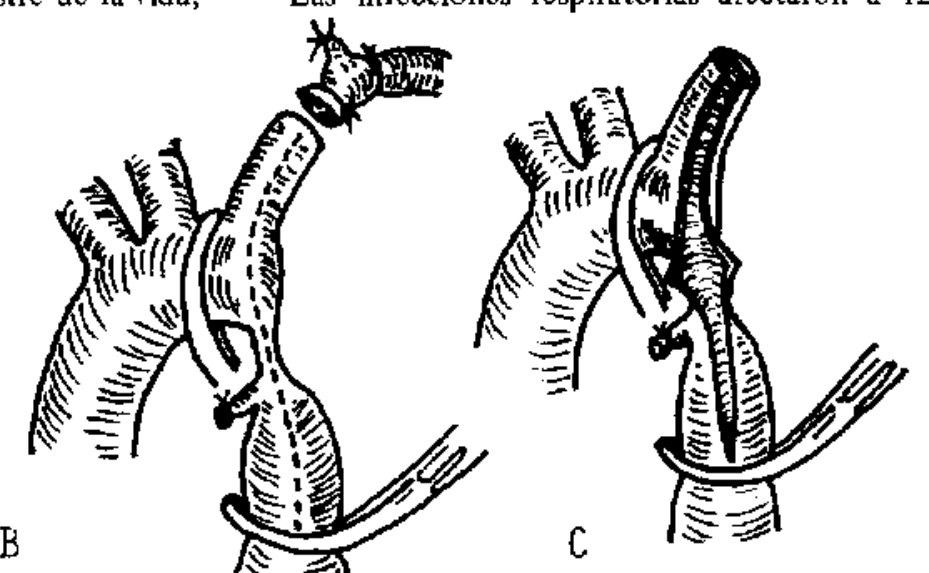

C

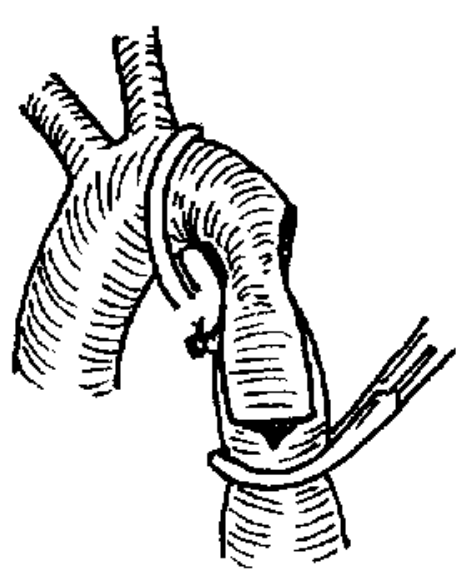

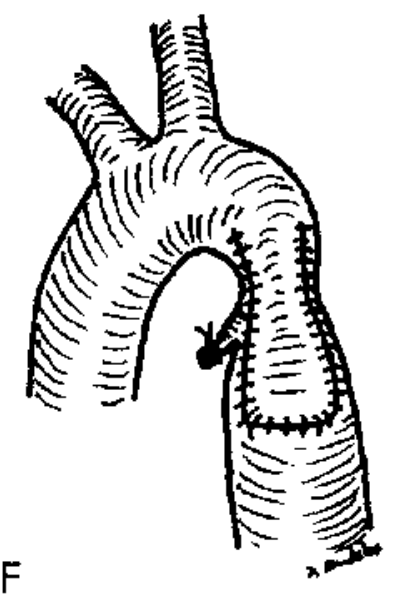

Figura 1: Técnica de plástia con arteria subclavia en reparación de coartación aórtica: A) Zona coartada a nivel de aorta. B) Ligadura de artería subclavia y vertebral izquierda. C) Apertusa de canal en la artesia subclavia. D) Colgajo de arteria subclavia, E) Colocación del colgajo en zona coartada. F) Sutura y resultado final. (Modificado de: Thibault. Arch. Surg. 110: 1095, 1975). 
(18\%) de los casos; en proporciones de $27 \%, 31 \%$ y $17 \%$ en $\operatorname{los} 3$ primeros grupos etarios descri. tos en el párafo anterior, no afectando a nif́os mayores de 12 meses.

La hemorragia digestiva acurrió en $6(9 \%)$ casos; no ocurrió en menores de 3 meses; en los siguientes grupos su frecuencia fue de $8 \%, 33 \%$ y $15 \%$ respectivamente. Este fenómeno se observó en la fase inicial de la experiencia, siendo ahora rara, debido al uso de antiácidos $y$ antihipertensivos.

Dos pacientes $(3 \%)$ fallecieron en el post-operatorio inmediato, ambos eran lactantes menores de 3 meses, por bronconeumonia en uno y bronconeumonia con sepsis en otro a $\operatorname{los} 6$ y 11 días respectivamente. En otras palabras la mortalidad operatoria en el primer trimestre de la vida fue de $10 \%$ y estuvo ausente en edades mayores.

Fue posible seguir a 47 pacientes ( $75 \%$ ) durante un promedio de 22 meses, rango de 1 mes a 7 años, con control clínico de pulsos y presión arterial: cuarenta y un pacientes siguen asintoma. tícos $(87 \%)$. En dos de ellos se registró hipertensión arterial persistente $(4,2 \%)$ : ambos habian sido operados a las edades de 1,5 y 3 meses, pesando 2.680 y 4.200 gramos respectivamente, el menor tenía, además, ductus y miocardiopatía hipertensiva, su hipertensión se detectó dos meses después de la cirugía y la "recoartación" fue documentada mediante estudio hemodiná. mico; se reoperó con anastomosis términoterminal, completando posteriomente 2 años sin síntomas. El segundo paciente no tenía cardiopatía asociada y se encontraba en estudio en el momento de la tabulación de estos datos (19 meses después de la operación).

La disminución del pulso en la extremidad superior izquierda fue constante en todos los pacientes operados. En un paciente se encontró evidencia de fallas de la irrigación de esa extremidad, consistentes en atrofia muscular y menor longitud $(3 \mathrm{~cm})$ que en la derecha. El paciente con seguimiento más largo (7 años) no tiene, sin embargo, fenómenos tróficos en la extremidad.

En el largo plazo fallecieron tres pacientes: 3 , 4 y 6 meses después de la operación, dos debido a infecciones respiratorias y uno durante cinigia con circulación extracorpórea para reparar una CIV con hipertensión pulmonar. Ninguno tenía evidencias de recoartación.

\section{COMENTARIO}

La corrección de la Co Ao con colgajo de arteria subclavia izquierda fue descrita por primera vez en $1966^{5}$. Desde entonces se ha utilizado con creciente frecuencia hasta llegar a ser la técnica de elección para la reparación de esta cardiopatía en el niño $0^{6,7,8}$. Sus riesgos inmediatos son la hemorragia y la crisis hipertensiva paradojal. La hipertensión arterial que observamos en nuestros pacientes ha sido descrita por otros y responde al uso de hipotensores como la reserpina; la mayor parte de los autores piensan que se debe a hiperreactividad del territotio arterial, después del restablecimiento del flujo aórtico distal normal ${ }^{1}$.

La mortalidad del procedimiento es de 10 a $24 \%$ en series extranjeras ${ }^{9,9}$. La baja mortalidad (3\%) en nuestra casuistica se compara favorable. mente con otras similares ${ }^{9}$. La causa de muerte coincide con lo publicado y se debe principalmente a infecciones respiratorias graves ${ }^{\mathrm{g}}$.

Se señala que la recoartación ocurre en $20 \%$ de los casos, generalmente antes de transcurridos 12 meses desde la cirugia. Se ha atribuido al hecho de dejar in situ tejido patológico de la zona de.coartación, cuyo curso natural sería hacia la involución y retracción, fenómeno que probablemente se completaria en este período. Esto explica que la recoartación ocurra casi siempre en pacientes menores de 3 meses sometidos a la técnica descrita ${ }^{8,10,11}$; En nuestra revisión los 2 pacientes que sufrieron recoartación se incluyen en ese rango de edad

En el seguimiento a largo plazo se ha observado disminución o ausencia de pulso arterial en la extremidad superior izquierda y con menos frecuencia trastornos tróficos, incluyendo disminución de la temperatura, atrofia muscular y acortamiento de la extremidad, atribuíbles a la ligadura de la arteria subclavia12,13. En nuestra revisión todos los pacientes mostraban disminución o ausencia de pulso, pero sólo uno trastornos tróficos.

De este análisis se puede concluir que esta plastía tiene baja mortalidad y buenos resultados alejados, sin secuelas invalidantes de la extremidad superior izquierda, a pesar de lo cual los resultados de ésta y otras experiencias sugieren la necesidad de revisar el empleo de este procedimiento en menores de 3 meses, pues estos niños muestran mayor incidencia de recoartación.

Sólo un estudio prospectivo y comparativo en este grupo de edad podría responder esta controversia.

\section{RESUMEN}

Se analizaron retrospectivamente los casos de 65 niños con coartación de aorta sometidos a piastía con colgajo de arteria subclavia, entre Noviembre de 1977 y Enero de 1985. El rango de edad de los pacientes fue de 7 días a 10 an os, 
$y$ el $58 \%$ tenian otra malformación cardiaca concomitante. La mortalidad operatoria global fue de $3 \%$, se debió a complicaciones respirato. rias y sucedió sólo en menores de 3 meses. En el seguimiento se detectó recoartación en 2 pacientes (ambos habían șido operados en el primer trimestre de vida). Sólo un paciente tuvo atrofia muscular y acortamiento de la extremidad como secuela de la ligadura de la arteria subclavia. La baja mortalidad que acarrea el procedimiento y la buena evolución alejada de los pacientes en que se ha usado, nos hacen considerarlo como una técnica de elección en niños con coartación aórtica.

\section{AGRADECIMIENTOS}

Nuestro agradecimiento a los doctores Helmuth Jaeger, Domingo Vicuña, Sonia Vesa y Gabriela Enríquez, quienes contribuyeron en la obtención de la información $\mathrm{y}$ al tratamiento de los pacientes incluidos en esta serie.

\section{REFERENCLAS}

1. Spencer F.C.: "Congenital Heart Disease" in Principles of Surgery. Seymour I. Schwarta. Third Edition Vol. II. Ed. y Mc. Graw-Hill Book Company, New York.

2. Parker F.B., Farrell B., Streeten D.H.: "Hypertensive mechanisms in coarctattion of the aorta". J. Thorac Cardiovasc. Surg. 80:568, 1980.

3. Tucker B.L., Staton R.E., Lindensmith G.G.: "Recurrent coarctation of the Thoracic aorta". Arch, Surg: 102: 556, 1971.

4. Fishmon N.H., Bronstein M.H., Berman W.: "Surgical management of severe aortic coarctation and interrupted aortic arch in neonates". I. Thorac-Cardiovasc. Surg. 71: 36, 1976.
5. Woldhausen J.A., Norhwold D.L.: "Repair of coarctation of the aorta with subclavian flap". J. Thorac. Cardiovasc. Surg. 51: 531, 1966.

6. Midgley F.M., Scott L.P., Perry L.W.: "Subclavian flap aortoplasty for treatment of coarctation in early infancy". J. Pediatr. Surg. 13: 265, 1978.

7. Pierce W.S., Waldhausen J.A., Beman W.: "Late results of the subclavian flap procedure in infants with coarctation of the thoracic aorta". Circulation Suppl. 1, 5B: 78, 1978.

8. Thibault W.N., Sperling D.R., Gazaniga A.B.: "Subclavian Artery Patch Angioplasty". Arch. Surg. 110: 1095, 1975

9. Hamilton D.f., Sandrasagra F.A., Donnolly R.J.: "Early and late results of aortoplasty with a left subclavian flap for coarctation of the aorta in infancy", J. Thorac. Cardiovasc. Surg. 75: 699, 1978.

10. Metzdorff M,T., Cobanogh A., Gnunkemeier G.L.: "Influence of age at operation on late results with subclavian fiap aortoplasty", J. Thorac. Cardjovasc. Surg. 89: 235,1985

11. Cabanogh A., Teply J.F., Khonoari S.: "Coarctation of the aorta under 3 month of age a critique of the subclavian flap operation". J. Thorac. Cardiovasc. Surg. 39: 128, 1985 .

12. Lodge F.A., Lamberti J.J., Goodman A.H.: "Vasculat consequences of subclavian artery transection for the treatment of Congenital Heart Disease". J. Thorac. Cardiovasc. Surg. B6: 18, 1983.

13. Todd P.J., Dongerfield P.H. Hamilion D.L.: "Late Affects on the left upper limb of subclavian flap aortoplasty". J. Thorac. Cardiovasc. Surg. 85: 678, 1983.

14. Norero C.V., Vargas N.C., Mayne D. V., Fernández $C .:$ "Cifras sencionales en población escolar chilena urbano". I Parte. Rev. Chil. Ped iatr. 51: 184, 1980.

15. Norero C.V., Vargas N.C., Mayne D. V., Monti A.F.: "Cifras sencionales en la población escolar chilena urbano". II Parte. Distribución por percentiles. Rev. Chil. Pediatr. 52: 467, 1981.

16. Scroggie S.A., Gallardo E.G., Montenegro P.H., Bondi P.H. " Medición sinullaneo Indirecta de la presión arterial en lactantes mediante tres métodos. Rev, Chil. Pediatr. 53: \$58, 1982. 